\title{
Liver Injury Associated with Metamizole Exposure: Features of an Underestimated Adverse Event
}

\author{
Sabine Weber ${ }^{1}$ (D) $\cdot$ Andreas Benesic $^{1,2,3} \cdot$ Jens Neumann $^{4} \cdot$ Alexander L. Gerbes $^{1}$ (D)
}

Accepted: 1 February 2021 / Published online: 27 February 2021

(c) The Author(s) 2021

\begin{abstract}
Introduction and Objective The potential of metamizole to cause drug-induced liver injury (DILI) has received increasing attention. We investigated the distinguishing features of a case series comprising 32 patients with suspected metamizoleinduced DILI.

Methods For the current analysis, 32 of 238 patients with DILI included in our prospective study on drugs potentially causing DILI were included. Diagnosis of DILI was based on expert opinion and RUCAM (Roussel Uclaf Causality Assessment Method) score and supported by an in vitro test using monocyte-derived hepatocyte-like cells.

Results Suspected metamizole-DILI was characterised by a female predominance, hepatocellular pattern of injury, high proportion of antinuclear antibody positivity, and predominance of eosinophilic cell infiltration and necrosis in the histopathological analysis. With 22\%, a high proportion of these metamizole-associated liver injury cases developed acute liver failure, which was characterised by a longer latency of metamizole use and more pronounced liver biochemistry abnormalities at onset and peak levels. Furthermore, jaundice was a common finding in the metamizole-associated liver injury cases with $66 \%$ presenting with peak bilirubin levels of $3 \mathrm{mg} / \mathrm{dL}$ or higher, which was associated with a worse outcome and a higher frequency of acute liver failure.

Conclusions Our analysis of a well-characterised DILI cohort further supports the potential of metamizole causing DILI and provides important features for the establishment of a signature pattern of liver injury observed in patients treated with metamizole.
\end{abstract}

Clinical Trial Registration ClinicalTrials.gov: NCT 02353455.

\section{Introduction}

Metamizole is an analgetic, antipyretic and spasmolytic drug that was patented in Germany in 1922. It has been banned from the market in the USA and the UK because of its side effects, mainly severe agranulocytosis $[1,2]$. However, it is still widely used in several countries and particularly in Germany the use has increased in recent years [3]. Yet, despite its use over several decades, only a few reports on

Sabine Weber

sabine.weber@med.uni-muenchen.de

1 Department of Medicine II, Liver Centre Munich, University Hospital, LMU Munich, Marchioninistr. 15, 81377 Munich, Bavaria, Germany

2 Krankenhaus GmbH Weilheim, Schongau, Germany

3 MetaHeps GmbH, Planegg, Bavaria, Germany

4 Institute of Pathology, Faculty of Medicine, LMU Munich, Munich, Bavaria, Germany liver damage caused by metamizole have been published until recently [4]. In the drug label in Germany, neither the deterioration of liver function tests nor acute liver failure (ALF) is listed as an adverse event. Considering the extensive hepatic metabolism of metamizole, mainly mediated by cytochrome P450 [5], its lipophilicity and the relatively high daily dose, all of which are known risk factors for druginduced liver injury (DILI) [6, 7], it is surprising that the hepatotoxic potential has not been a topic for pharmacovigilance until now. Interestingly, Sebode et al. reported 23 cases of metamizole-induced DILI recently, which corresponded to $15 \%$ of all the DILI cases included in their centre [8]. The majority of the cases presented with a hepatocellular type of injury, nearly $50 \%$ of patients fulfilled Hy's law criteria, meaning alanine aminotransferase (ALT) $>3 \times$ the upper limit of normal (ULN) and total bilirubin (TBIL) $>2 \times$ ULN, and $9 \%$ of the patients developed ALF [8].

Drug-induced liver injury is a diagnosis of exclusion, based on ruling out other causes for liver injury [9]. Because of the lack of specific biomarkers or diagnostic tests, 


\section{Key Points}

The analgetic drug metamizole was found to have probably caused drug-induced liver injury in $13 \%$ of all cases from our study cohort on suspected drug-induced acute liver injury, although other aetiologies including concomitantly administered products cannot be excluded as the cause of liver injury in some cases.

These metamizole-associated liver injuries are characterised by a hepatocellular type of injury, positive antinuclear antibodies, histopathological signs of inflammatory infiltrates with mainly eosinophilic cells as well as high proportions of moderate-to-severe necrosis.

A high proportion of patients with metamizole-associated DILI developed acute liver failure. These cases of liver failure were characterised by a latency of 2-3 months from metamizole intake to the onset of liver injury and high levels of transaminases, bilirubin and international normalised ratio both at onset and at respective peak levels.

Cholestatic forms of metamizole-associated DILI occur and are associated with a worse outcome.

adjudication of the culprit drug is based on causality assessment scores, mainly the RUCAM (Roussel Uclaf Causality Assessment Method) score and expert opinion [6, 10]. However, relying on the RUCAM may not always be practical, as RUCAM comprises previously described DILI potential and reaction to re-exposure [11]. Thus, especially in the setting of polypharmacy, drugs that have formerly not been known to cause DILI will not be associated with liver injury, causing a bias towards underestimation of their potential to cause liver injury. To further investigate the role and features of metamizole in DILI, we analysed the clinical characteristics of patients with suspected metamizole-induced acute liver injury and compared the distinctive features of more severe metamizole-DILI cases.

\section{Methods}

\subsection{Patient Cohort}

The data from 383 patients who were referred to the University Hospital Munich with acute liver injury and recruited for our ongoing prospective study on the effects of potentially hepatotoxic drugs (ClinicalTrials.gov: NCT 02353455) between March 2013 and July 2020 were analysed. Included in this study are healthy donors, patients prior to receiving therapy with drugs with DILI potential, patients with acute liver injury and DILI suspicion, as well as patients with acute liver injury as a result of other causes. Written informed consent was obtained from each patient. The study protocol conforms to the ethical guidelines of the Declaration of Helsinki and was approved by the Ethics Committee of the Faculty of Medicine, LMU Munich (project number 55-13). For the current analysis, 32 patients who had consumed metamizole were selected.

\subsection{Definition, Diagnosis and Classification of DILI}

Acute liver injury was defined according to the consensus criteria from 2011: (a) ALT $\geq 5 \times$ ULN, (b) alkaline phosphatase activity $\geq 2 \times$ ULN, or (c) ALT $\geq 3 \times$ ULN and TBIL $\geq 2 \times$ ULN [12]. The ULN for aspartate aminotransferase (AST) activity and ALT were $35 \mathrm{U} / \mathrm{L}$ for women and $50 \mathrm{U} / \mathrm{L}$ for men, the ULN for alkaline phosphatase was $105 \mathrm{U} / \mathrm{L}$ for women and $130 \mathrm{U} / \mathrm{L}$ for men and the ULN for TBIL was $1.2 \mathrm{mg} / \mathrm{dL}$. The type of liver injury was classified using the $R$ ratio values, (ALT/ULN)/(alkaline phosphatase/ ULN), with $R \geq 5$ defining a hepatocellular injury, $R \leq 2$ a cholestatic injury and $2<R<5$ defining a mixed-type injury [13].

The diagnosis of DILI was based on clinical and laboratory findings, the RUCAM score [14], a causality assessment by the treating physician and expert opinion [15], as well as an in vitro test established in our centre using monocyte-derived hepatocyte-like cells (MH cells) [16-18]. The interpretation of the RUCAM score is: $<0$, drug is excluded as the cause; $1-2$, unlikely; $3-5$, possible; $6-8$, probable; and $>8$, highly probable. Regarding the RUCAM score for metamizole, 1 point was given for the item 'known hepatoxicity', as liver injury is not listed as an adverse event in the drug label of metamizole but has been described in the literature $[4,8]$. A causality assessment by the treating physician was conducted and expert opinion from consulting hepatologists from our centre was used by consideration of all clinical data available, the course of events and by using the LiverTox database [19]. The assessment of causality was performed independently from the $\mathrm{MH}$ cell test result.

Blood samples for $\mathrm{MH}$ cell testing were acquired within 4 weeks after the onset of liver injury. Monocyte-derived hepatocyte-like cell generation and testing were performed as described previously [20]. Briefly, monocytes were isolated from patients' blood samples and cultivated under serum-free conditions for 10 days, generating cells with some hepatocyte features such as cytochrome P450 activities. These cells, $\mathrm{MH}$ cells, are incubated for 48 hours in 96-well plates using $1 \times$ maximum concentration $\left(C_{\max }\right)$ and $10 \times C_{\max }$ of the implicated drugs the respective patient had consumed. For the current analysis, MH cell 
testing was performed for every patient and metamizole was tested in $94 \%$ of the patients included along with the concomitant medications used by the respective patients. Metamizole was used in concentrations of $50 \mu \mathrm{M}$ and 500 $\mu \mathrm{M}$, which equals $1 \times C_{\max }$ and $10 \times C_{\max }$ of this drug. The lowest amount of cells used for a single test is 10,000 cells per well. All drugs are solved in a standardised medium (DMEM/HAM-F12 containing penicillin/streptomycin and 2 L-glutamine). Then, toxicity is measured with a standardised algorithm based on the release of lactate dehydrogenase in the supernatant and cell lysate. Results are normalised to a negative (0\%) and positive control (100\%), meaning lysis with $1 \%$ TWEEN $^{\circledR} 20$ (polyethylene glycol sorbitan monolaurate). To compensate for variance in seeding density, toxicity values are divided by two standard deviations (ULN) of the individual controls. The vector graphic demonstrating $\mathrm{MH}$ cell test results was created using Prism 8, Version 8.4.3.

The severity of the DILI episode was categorised as mild (1), moderate (2), severe (3) or fatal (4; death or the need for liver transplantation because of liver failure) according to the 2011 criteria of an international DILI expert group [12]. Acute liver failure was defined according to the American Association for the Study of Liver Diseases: (1) absence of pre-existing liver disease, (2) coagulopathy with an INR $\geq 1.5$ in the absence of oral anticoagulants and (3) hepatic encephalopathy [21]. 'Hy's law' criteria were fulfilled if ALT was $\geq 3 \times$ ULN and TBIL was $\geq 2 \times$ ULN, defining a subgroup of patients with DILI with a $10 \%$ risk of fatality or need of liver transplantation [22].

\subsection{Data Collection}

At enrolment, a thorough medical history was taken, including previous diseases, symptoms associated with liver injury, co-morbidities as well as current or previous medications. Each patient's age, sex, ethnicity, height, weight and relevant data from clinical investigations were recorded. A thorough hepatological work-up, including liver function tests, serological studies for hepatitis A, B, C, D, and E, cytomegalovirus, Epstein-Barr virus and herpes simplex virus as well as autoantibody screening was performed, and results were extracted from the medical records. The presence of antinuclear antibodies (ANA) and other liver-specific antibodies was tested using an indirect immunofluorescence assay kit, Mosaic Basic Profile 3 (Euroimmun, Lübeck, Germany). The immunofluorescence assay was performed with an initial dilution of 1:100. In addition to serological testing, hepatitis E polymerase chain reaction was performed in 50\% of the cases. Whenever a liver biopsy was conducted during the diagnostic work-up, the results were extracted from the pathological report.

\subsection{Statistical Analysis}

Statistical analyses were performed using SPSS, Version 26.0.0.1 (IBM, Armonk, NY, USA). After testing for normal distribution, parametric or non-parametric tests (Chi-square test, Fisher's exact test or Mann-Whitney $U$ test) were applied, $p \leq 0.05$ was considered statistically significant.

\section{Results}

\subsection{Clinical Characteristics and Outcome of Patients with Suspected Metamizole-Induced DILI}

From our cohort of 383 patients with acute liver injury who were included in our prospective study on potential DILI drugs owing to the initial suspicion of DILI, 238 patients were diagnosed with DILI. Out of those 238 patients, we identified 32 patients with suspected metamizole-induced DILI (13\%). All of these patients had a concomitant medication: non-steroidal antirheumatic drugs were used in ten (31\%) cases, antibiotics also in ten (31\%) cases, anaesthetics in three (9\%) cases, herbals in two (6\%) cases and others in seven $(22 \%)$ cases. For an overview of the concomitant medication, see Table 1 of the Electronic Supplementary Material (ESM). In all of the patients, RUCAM for metamizole was $\geq 3$, meaning that metamizole was possibly, probably or highly probably the causative agent for the DILI episode. Adjudication by the treating physician revealed metamizoleinduced DILI as the most likely diagnosis in eight of the patients with DILI (25\%), while in the remaining 24 patients (75\%) an association with metamizole as the causative agent was assessed as the most likely diagnosis but could not be proven because of the intake of concomitant medication with a compatible time to onset. The clinical characteristics of the patients with suspected metamizole-induced DILI are summarised in Table 1. Sixty-nine percent of the patients were female and the median age at diagnosis was 41 years. In the majority of cases, the patients presented with a hepatocellular type of injury $(n=26,81 \%)$. The median RUCAM score both for metamizole and the concomitant medication with the highest RUCAM was 5 (range 3-9). Anti-nuclear antibodies were found in $72 \%(n=23)$ of the patients, while $34 \%(n=11)$ presented with positive antimitochondrial antibodies (AMA). Median immunoglobulin G levels were within the range of normal $(13.1 \mathrm{~g} / \mathrm{L}[5.3-24.3 \mathrm{~g} / \mathrm{L}]$; ULN: $16 \mathrm{~g} / \mathrm{L}$ ), none of the patients were positive for other liver-specific autoantibodies, such as anti-smooth muscle antibodies, liver-kidney microsomal antibodies, or soluble liver antigen, and none of the patients had a persistent liver injury or relapse, ruling out autoimmune hepatitis or chronic autoimmune biliary disease. The median follow-up was 5 
months, which ensured that relapse of liver injury did not occur after the acute episode of DILI.

Eight patients received corticosteroid treatment (25\%), which was given at the discretion of the treating physician. Discontinuation of corticosteroids was possible in every patient. Most of the patients $(n=31,94 \%)$ were in remission at the end of the follow-up, while two patients $(6 \%)$ needed an emergency liver transplant because of ALF.

A liver biopsy was performed in 20 of the 32 patients (63\%). The histopathological characteristics found in the liver specimen analysis are provided in Table 2 of the ESM. Inflammatory infiltrates were reported for every patient with a predominant infiltration with eosinophilic cells ( $n$ $=17,85 \%)$. Further, in the majority of cases necrosis $(n=$ $17,85 \%)$ of mostly moderate to pronounced intensity ( $n=$ $12 / 17,71 \%$ ) was observed. Infiltration with plasma cells was found in 12 patients $(60 \%)$. In four cases, a low-grade fibrosis was detected, while none of the patients presented with advanced fibrosis. Representative pictures of eosinophilic

Table 1 Clinical characteristics in patients with suspected metamizole-induced acute liver injury $(n=32)$

\begin{tabular}{ll}
\hline Age (years) & $41(19-79)$ \\
Body mass index $\left(\mathrm{kg} / \mathrm{m}^{2}\right)$ & $23.3(18.1-37.9)$ \\
Female sex & $22(69 \%)$ \\
RUCAM (metamizole) & $5(3-9)$ \\
Highest RUCAM (concomitant medication) & $5(3-9)$ \\
Latency metamizole (days) & $42(2-674)$ \\
Daily dosage metamizole $(\mathrm{mg})^{\mathrm{a}}$ & $1250(40-4000)$ \\
Pattern of injury & $26(81 \%)$ \\
Hepatocellular & $5(16 \%)$ \\
Mixed & $1(3 \%)$ \\
Cholestatic & \\
ANA & $23(72 \%)$ \\
AMA & $11(34 \%)$ \\
IgG (g/L, ULN 16 g/L) & $13.1(5.3-24.3)$ \\
Severity according to Aithal et al. & \\
Corticosteroid treatment & $2(1-4)$ \\
Acute liver failure & $8(25 \%)$ \\
Need for high-urgency liver transplantation & $7(22 \%)$ \\
Positive re-challenge with & $2(6 \%)$ \\
Metamizole & $3(9 \%)$ \\
Metamizole and concomitant medication & $4(13 \%)$ \\
MH cell test positive for metamizole & $24(75 \%)$ \\
\hline
\end{tabular}

Categorical variables are presented as number and percentage $[n$ (\%)]. Continuous variables are presented as median (range)

$A M A$ antimitochondrial antibodies, $A N A$ antinuclear antibodies, $I g G$ immunoglobulin G, $\mathrm{MH}$ cell test monocyte-derived hepatocyte-like cell test, RUCAM Roussel Uclaf Causality Assessment Method, ULN upper limit of normal

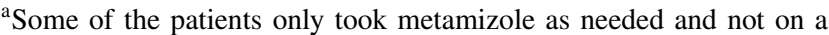
regular daily basis. For those patients, the average daily dose according to the weekly intake was calculated

${ }^{\mathrm{b}}$ Aithal et al. Clin Pharmacol Ther. 2011;89(6):806-15 cell infiltration and necrosis in a liver biopsy from a patient with DILI from our cohort are shown in Fig. 1a-d. Seven patients had a positive re-challenge, meaning that they had a second episode of acute liver injury after unintentional re-exposure to metamizole alone $(n=3,9 \%)$ or a combination of metamizole and the respective comedications $(n=$ $4,13 \%)$.

\subsection{Subgroup Analysis: Comparison of Clinical and Histological Characteristics in Patients with Suspected Metamizole-Induced Liver Injury in Relevance to a Causality Assessment Based on RUCAM Scoring}

If patients with RUCAM $\geq 6$ for metamizole (metamizoleinduced DILI probable or highly probable; $n=12$ ) and RUCAM 3-5 (metamizole-induced DILI possible; $n=20$ ) were compared, no significant differences regarding clinical, laboratory and histopathological results were observed (Table 2). Age, sex distribution, the predominantly hepatocellular type of injury, the level of liver function test abnormalities both at the onset of disease and at peak value, severity and outcome were similar between both groups. The only difference was that patients with RUCAM $<6$ presented with higher rates of AMA positivity ( $45 \%$ vs $17 \%, p$ $=0.024)$. Histopathological analysis revealed that cholestasis was observed less often in patients with RUCAM $\geq 6$ for metamizole ( $10 \%$ vs $50 \%$ ); however, this difference was not statistically significant $(p=0.051)$. Other histological patterns with moderately strong inflammatory infiltrates and moderate to pronounced necrosis were comparable between patients with RUCAM $\geq 6$ and RUCAM $<6$ (Table 3 of the ESM). A detailed overview of the characteristics of the individual patients with suspected metamizole-induced DILI cases and high RUCAM scores for metamizole is provided in Table 4 of the ESM.

\subsection{Subgroup Analysis: Comparison of Clinical and Histological Characteristics in Patients with Suspected Metamizole-Induced Liver Injury in Relevance to a Causality Assessment Based on MH Cell Test Results}

Reliability of the RUCAM for a causality assessment is limited [11], especially in polymedicated patients. This is also illustrated in the current cohort: eight patients $(25 \%)$ had a RUCAM of 6 or higher for both metamizole and at least one of the concomitant medications, while another six patients (19\%) had RUCAM scores of 6 or higher for at least one of the concomitant drugs but not metamizole. In addition to expert opinion and RUCAM scoring as a gold standard for establishing the diagnosis, the causality assessment was supported by the MH cell test, an in vitro test system established 
Table 2 Comparison of clinical characteristics and outcome in patients with suspected metamizole-induced liver injury and RUCAM $\geq 6$ vs RUCAM $<6$ for metamizole

\begin{tabular}{|c|c|c|c|}
\hline & $\begin{array}{l}\text { RUCAM (metamizole) } \geq 6 \\
n=12(37.5 \%)\end{array}$ & $\begin{array}{l}\text { RUCAM (metamizole) }<6 \\
n=20(62.5 \%)\end{array}$ & $p$ value \\
\hline Age (years) & $55(19-79)$ & $38(19-61)$ & 0.17 \\
\hline Body mass index $\left(\mathrm{kg} / \mathrm{m}^{2}\right)$ & $23.2(18.1-30.6)$ & $24.2(18.9-37.9)$ & 0.41 \\
\hline Female sex & $10(77 \%)$ & $14(61 \%)$ & 0.56 \\
\hline Latency metamizole (days) & $46(2-370)$ & $42(8-674)$ & 0.55 \\
\hline Daily dosage metamizole $(\mathrm{mg})^{\mathrm{a}}$ & $1000(71-4000)$ & $1500(500-3000)$ & 0.36 \\
\hline $\begin{array}{l}\text { Pattern of injury } \\
\text { Hepatocellular } \\
\text { Mixed } \\
\text { Cholestatic }\end{array}$ & $\begin{array}{l}\quad 9(75 \%) \\
2(17 \%) \\
1(8 \%)\end{array}$ & $\begin{array}{l}17(85 \%) \\
3(15 \%) \\
0(0 \%)\end{array}$ & 0.41 \\
\hline Peak ALT & $29.8(5.9-105.5)$ & $45.3(6.9-105.9)$ & 0.17 \\
\hline Peak AST & $12.5(3.1-59.4)$ & $35.4(1.3-162.2)$ & 0.08 \\
\hline Peak ALP & $2.0(1.0-7.8)$ & $1.6(1.0-4.3)$ & 0.55 \\
\hline Peak TBIL & $5.8(0.7-27.9)$ & $11.2(0.5-29.9)$ & 0.66 \\
\hline Peak INR & $1.3(0.9-6.6)$ & $1.3(0.9-8.0)$ & 0.27 \\
\hline Peak MELD & $14(6-40)$ & $20(6-40)$ & 0.27 \\
\hline $\operatorname{IgG}(\mathrm{g} / \mathrm{L}, \mathrm{ULN} 16 \mathrm{~g} / \mathrm{L})$ & $11.4(5.3-17.6)$ & $13.5(7.0-24.3)$ & 0.37 \\
\hline ANA positivity & $11(92 \%)$ & $12(60 \%)$ & 0.054 \\
\hline AMA positivity & $2(17 \%)$ & $9(45 \%)$ & $\mathbf{0 . 0 2 4} *$ \\
\hline Severity according to Aithal et al. ${ }^{\mathrm{b}}$ & $2(1-4)$ & $2(2-4)$ & 0.92 \\
\hline Jaundice (TBIL > 3 mg/dL) & $8(67 \%)$ & $13(65 \%)$ & 0.92 \\
\hline Coagulopathy (INR $>1.5$ ) & $4(33 \%)$ & $8(40 \%)$ & 0.71 \\
\hline Hy's law criteria & $9(75 \%)$ & $16(80 \%)$ & 0.74 \\
\hline Acute liver failure & $2(17 \%)$ & $5(25 \%)$ & 0.58 \\
\hline Corticosteroid treatment & $3(25 \%)$ & $5(25 \%)$ & 1.00 \\
\hline High-urgency liver transplantation & $1(8 \%)$ & $1(5 \%)$ & 0.69 \\
\hline $\begin{array}{l}\text { Re-exposure with } \\
\text { Metamizole alone } \\
\text { Metamizole and concomitant medication }\end{array}$ & $\begin{array}{l}2(17 \%) \\
3(25 \%)\end{array}$ & $\begin{array}{l}1(5 \%) \\
1(5 \%)\end{array}$ & 0.11 \\
\hline MH cell test positive for metamizole & $10(83 \%)$ & $14(70 \%)$ & 0.49 \\
\hline
\end{tabular}

Categorical variables are presented as number and percentage $[n(\%)]$. Continuous variables are presented as median (range). Laboratory values are presented as $\times$ ULN

$A L P$ alkaline phosphatase, $A L T$ alanine aminotransferase, $A M A$ antimitochondrial antibodies, $A N A$ antinuclear antibodies, $A S T$ aspartate aminotransferase, $I g G$ immunoglobulin G, INR international normalised ratio, MELD Model of End Stage Liver Disease, $M H$ cell test monocytederived hepatocyte-like cell test, RUCAM Roussel Uclaf Causality Assessment Method, TBIL total bilirubin, $U L N$ upper limit of normal

*Statistical significance $(p \leq 0.05)$

${ }^{a}$ Some of the patients only took metamizole as needed and not on a regular daily basis. For those patients, the average daily dose according to the weekly intake was calculated

${ }^{\mathrm{b}}$ Aithal et al. Clin Pharmacol Ther. 2011;89(6):806-15

in our centre and validated in patients with re-exposure previously [17]. The MH cell test was performed for every patient in this cohort and metamizole was tested for in 30/32 (94\%) of the cases. The MH cell test showed toxicity against metamizole in 24 of those 30 patients (75\%). An overview of the individual test results for metamizole is shown in Fig. 1 of the ESM. Patients with positive MH cell test results for metamizole were more likely to present with a hepatocellular type of injury ( $88 \%$ vs $50 \%, p=0.048)$. Otherwise, no significant differences were observed regarding clinical or laboratory characteristics (Table 3). Histopathological patterns were also similar in patients who tested negative and positive for metamizole in the MH cell test (Table 3 of the ESM).

A special focus was placed on the 14 patients who presented with a RUCAM for the concomitant medication of at least 6 . As stated above, eight of those patients also had a RUCAM for metamizole of 6 or higher. An overview over the individual patients' MH cell test results for metamizole and the concomitant drugs is given in Table 5 of the ESM. 
Fig. 1 Histology of liver biopsy. Liver biopsy revealed moderate portal and lobular hepatitis with liver cell drop out, confluent necrosis and mild fibrosis (a, haematoxylin and eosin stain, 25 -fold). In a larger magnification $(\mathbf{b}$, haematoxylin and eosin stain, 100-fold), lymphohistiocytic infiltrates with scattered eosinophils and apoptosis of liver cells could be obtained. In c (haematoxylin and eosin stain, 200-fold), aggregated eosinophil granulocytes and in $\mathbf{d}$ (haematoxylin and eosin stain, 200-fold) apoptotic bodies ('Councilman bodies') are shown
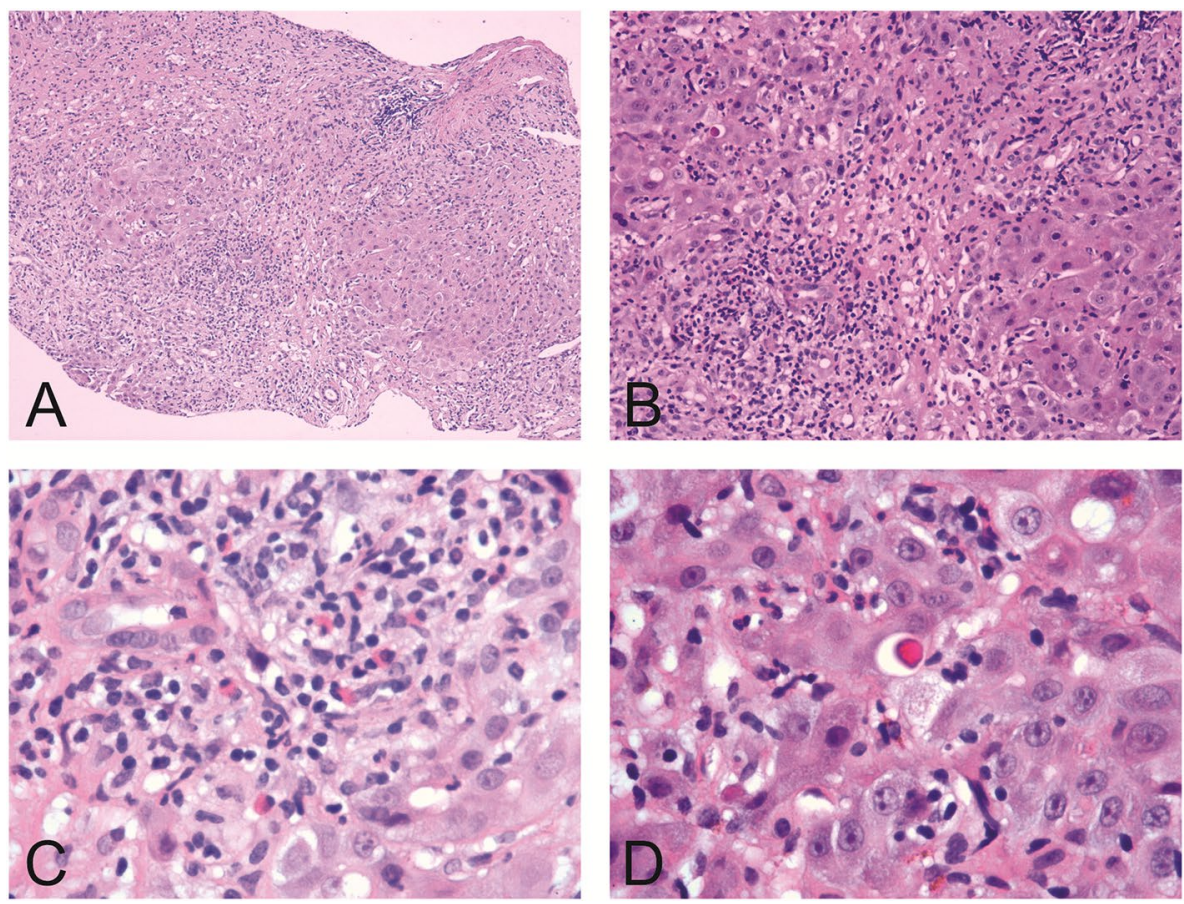

The $\mathrm{MH}$ cell test results showed toxicity of metamizole but not of the comedication in seven $(50 \%)$ cases and of a combination of metamizole and the comedication in three (21\%) cases. In four cases (29\%), the $\mathrm{MH}$ cell test was negative for both metamizole and the co-administered drug.

In addition to testing patients with suspected metamizole-DILI, MH cell testing has also been performed on a variety of control subjects: (a) healthy controls $(n=$ 8), (b) patients with metamizole intake and acute liver injury caused by conditions other than DILI $(n=18)$, (c) patients with DILI without metamizole intake $(n=$ 17) and (d) patients with metamizole treatment but DILI due to another drug $(n=39)$. Regarding the healthy controls, half had been exposed to metamizole $(n=4)$. The test was negative in seven of all eight healthy controls (88\%) and three of the four $(75 \%)$ healthy controls with prior metamizole intake. In 15 out of the 18 patients with intake of metamizole but liver injury not associated with DILI, the test showed negative results (83\%). Regarding DILI patients without a prior intake of metamizole, $\mathrm{MH}$ cell testing yielded negative results in $34 / 39$ cases $(87 \%)$. In patients with DILI with prior metamizole therapy but in whom liver injury was highly likely associated with another drug $(n=17)$, the MH cell test gave negative results in 16 cases $(94 \%)$. Positive results for the concomitant medication were observed in 16 of those 17 patients. One case had a positive result for both metamizole and the concomitant herbal drug and in one case the MH cell test was negative for both metamizole and the concomitant medication.

\subsection{Subgroup Analysis: Comparison of Clinical and Histological Characteristics in Patients with Suspected Metamizole-Induced Liver Injury According to Jaundice}

To focus on the more distinctive features of severe cases among all of the 32 cases of suspected metamizole-induced DILI, a subgroup analysis was performed comparing patients with DILI who developed a clinically relevant jaundice, i.e., peak TBIL $>3 \mathrm{mg} / \mathrm{dL}$, with patients without clinically relevant jaundice (Table 4). Regarding the basic clinical parameters, jaundiced patients with DILI-developed liver injury with a longer latency from the start of metamizole intake (58 vs 31 days, $p=0.039$ ) presented with higher peak levels of ALT, AST, TBIL and INR, as well as higher MELD scores both at the onset of liver injury and at peak levels. Severity according to the 2011 criteria [12] was significantly higher in jaundiced patients (severe [3] vs moderate [1], $p$ $=0.002$ ). Moreover, coagulopathy as well as Hy's law positivity was observed more often in jaundiced patients. This also translated into a higher occurrence of ALF, which was observed in $33 \%$ of the jaundiced patients but for none of the non-jaundiced patients $(p=0.03)$. Two of the jaundiced patients required high-urgency liver transplantation (10\% vs $0 \%, p=0.23)$. A liver biopsy was performed in the majority of patients with jaundice ( $n=15,71 \%$ ), and in $46 \%$ of the patients without jaundice ( $n=5$; Table 6 of the ESM). Interestingly, the proportion of patients presenting with steatosis was significantly higher in non-jaundiced patients $(60 \%$ vs $7 \%, p=0.034$ ) while cholestasis (mainly hepatocellular and 
Table 3 Comparison of clinical and laboratory features in patients with DILI and the intake of metamizole and positive or negative $\mathrm{MH}$ cell test results for metamizole

\begin{tabular}{|c|c|c|c|}
\hline & $\begin{array}{l}\text { Positive MH cell test } \\
\text { for metamizole } \\
n=24(75 \%)\end{array}$ & $\begin{array}{l}\text { Negative MH cell test } \\
\text { for metamizole } \\
n=6(25 \%)\end{array}$ & $p$ value \\
\hline Age (years) & $39(19-64)$ & $45(34-79)$ & 0.40 \\
\hline Body mass index $\left(\mathrm{kg} / \mathrm{m}^{2}\right)$ & $23.2(17.6-37.9)$ & $24.1(18.1-25.9)$ & 0.81 \\
\hline Female sex & $17(71 \%)$ & $4(67 \%)$ & 0.84 \\
\hline RUCAM (metamizole) & $5(3-9)$ & $5(4-7)$ & 0.49 \\
\hline Highest RUCAM (concomitant medication) & $5(3-9)$ & $6(5-6)$ & 0.53 \\
\hline Latency metamizole (days) & $46(2-674)$ & $41(15-82)$ & 0.64 \\
\hline Daily dosage metamizole $(\mathrm{mg})^{\mathrm{a}}$ & $1250(71-3000)$ & $1500(500-4000)$ & 0.77 \\
\hline $\begin{array}{l}\text { Pattern of injury } \\
\text { Hepatocellular } \\
\text { Mixed } \\
\text { Cholestatic }\end{array}$ & $\begin{aligned} & 21(87.5 \%) \\
3 & (12.5 \%) \\
0 & (0 \%)\end{aligned}$ & $\begin{array}{l}\quad 3(50 \%) \\
2(33 \%) \\
1(17 \%)\end{array}$ & 0.048* \\
\hline Severity according to Aithal et al. ${ }^{\mathrm{b}}$ & $2(1-4)$ & $2(2-3)$ & 0.40 \\
\hline Peak ALT & $37.4(5.9-105.5)$ & $24.1(7.6-105.9)$ & 0.43 \\
\hline Peak AST & $29.2(3.1-162.2)$ & $18.1(1.3-69.4)$ & 0.49 \\
\hline Peak ALP & $1.7(1.0-4.3)$ & $2.1(1.0-7.8)$ & 0.40 \\
\hline Peak TBIL & $5.8(0.5-29.9)$ & $6.0(0.8-23.1)$ & 0.71 \\
\hline Peak INR & $1.3(0.9-8.0)$ & $1.2(0.9-1.7)$ & 0.33 \\
\hline MELD at onset of liver injury & $12(6-30)$ & $12(6-24)$ & 0.72 \\
\hline Peak MELD & $14(6-40)$ & $15(6-24)$ & 0.89 \\
\hline Jaundice (TBIL > $3 \mathrm{mg} / \mathrm{dL}$ ) & $15(63 \%)$ & $4(67 \%)$ & 0.85 \\
\hline Coagulopathy (INR > 1.5) & $9(38 \%)$ & $1(17 \%)$ & 0.33 \\
\hline Hy's law criteria & $18(75 \%)$ & $5(83 \%)$ & 0.67 \\
\hline Acute liver failure & $5(21 \%)$ & $1(17 \%)$ & 0.82 \\
\hline Corticosteroid treatment & $6(25 \%)$ & $2(33 \%)$ & 0.68 \\
\hline High-urgency liver transplantation & $2(8 \%)$ & $0(0 \%)$ & 0.66 \\
\hline
\end{tabular}

MH cell test results for metamizole were available for $30(94 \%)$ patients. Categorical variables are presented as number and percentage of the respective group size $[n(\%)]$. Continuous variables are presented as median (range). Laboratory values are presented as $\times$ ULN

ALP alkaline phosphatase, ALT alanine aminotransferase, AST aspartate aminotransferase, DILI druginduced liver injury, INR international normalised ratio, MELD Model of End Stage Liver Disease, $M H$ cell test monocyte-derived hepatocyte-like cell test, RUCAM Roussel Uclaf Causality Assessment Method, TBIL total bilirubin, $U L N$ upper limit of normal

* Statistical significance $(p \leq 0.05)$

${ }^{\text {a }}$ Some of the patients only took metamizole as needed and not on a regular daily basis. For those patients, the average daily dose according to the weekly intake was calculated

${ }^{b}$ Aithal et al. Clin Pharmacol Ther. 2011;89(6):806-15 canalicular) and fibrosis were only observed in liver specimens of jaundiced patients (40\% vs $0 \%, p=0.09$; and $27 \%$ vs $0 \%, p=0.20$, respectively).

\subsection{Subgroup Analysis: Subgroup Analysis of Patients with ALF}

Seven of the 32 patients (22\%) with suspected metamizole-induced DILI presented with ALF. Concomitant medications used by these patients were ibuprofen $(n=4)$, cefuroxime $(n=4)$, sevoflurane $(n=3)$, propofol $(n=2)$, diclofenac $(n=2)$, acetylsalicylic acid, amoxicillin/clavulanic acid, ampicillin/sulbactam, ciprofloxacin, dienogest/ ethinylestradiol, pantoprazole, paracetamol, valsartan and zopiclone (each $n=1$ ). Patients who developed ALF had a longer latency between the initiation of metamizole and the onset of liver injury ( 80 vs 34 days, $p=0.033$ ) and higher levels of AST, TBIL, INR and MELD both at the onset of liver injury and at the respective peak levels as well as higher ALT levels at onset (Table 5). Patients with ALF also had a higher severity according to the 2011 criteria [12] and were more likely to present with jaundice or coagulopathy, while there was no significant difference in Hy's law positivity (Table 5). In accordance with the higher severity of those cases, the proportion of patients needing high-urgency liver transplantation was also significantly 
Table 4 Comparison of clinical characteristics and outcome in patients with suspected metamizole-induced liver according to the development of jaundice

\begin{tabular}{|c|c|c|c|}
\hline & $\begin{array}{l}\text { Jaundiced patients } \\
\left(\mathrm{TBIL}_{\text {peak }}>3 \mathrm{mg} / \mathrm{dL}\right) \\
n=21(66 \%)\end{array}$ & $\begin{array}{l}\text { Non-jaundiced patients } \\
\left.\text { (TBIL }_{\text {peak }} \leq 3 \mathrm{mg} / \mathrm{dL}\right) \\
n=11(34 \%)\end{array}$ & $p$ value \\
\hline Age (years) & $34(19-79)$ & $53(28-61)$ & 0.10 \\
\hline Body mass index $\left(\mathrm{kg} / \mathrm{m}^{2}\right)$ & $23.7(18.1-37.9)$ & $22.9(19.1-30.6)$ & 0.49 \\
\hline Female sex & $16(70 \%)$ & $8(62 \%)$ & 0.62 \\
\hline RUCAM (metamizole) & $5(3-8)$ & $5(3-9)$ & 0.79 \\
\hline Highest RUCAM (concomitant medication) & $6(3-9)$ & $5(4-8)$ & 0.46 \\
\hline Latency metamizole (days) & $58(2-674)$ & $31(2-55)$ & $0.039 *$ \\
\hline Daily dosage metamizole $(\mathrm{mg})^{\mathrm{a}}$ & $1000(71-4000)$ & $1500(500-3000)$ & 0.15 \\
\hline $\begin{array}{l}\text { Pattern of injury } \\
\text { Hepatocellular } \\
\text { Mixed } \\
\text { Cholestatic }\end{array}$ & $\begin{array}{l}17(81 \%) \\
3(14 \%) \\
1(5 \%)\end{array}$ & $\begin{array}{l}9(82 \%) \\
2(18 \%) \\
0(0 \%)\end{array}$ & 0.74 \\
\hline Peak ALT & $47.5(5.9-105.9)$ & $27.1(6.9-62.0)$ & $0.009 *$ \\
\hline Peak AST & $38.1(3.1-104.0)$ & $11.4(1.3-162.2)$ & $0.008^{*}$ \\
\hline Peak ALP & $1.8(1.0-7.8)$ & $1.6(1.0-2.5)$ & 0.10 \\
\hline Peak TBIL & $19.8(2.6-29.9)$ & $0.8(0.5-2.8)$ & $0.000 *$ \\
\hline Peak INR & $1.5(0.9-8)$ & $1.0(0.9-1.3)$ & $0.000 *$ \\
\hline ALT onset & $42.5(3.8-105.5)$ & $27.1(6.9-62.0)$ & 0.07 \\
\hline AST onset & $33.9(2.5-104.0)$ & $10.5(1.1-32.3)$ & $0.001 *$ \\
\hline ALP onset & $1.6(0.9-5.8)$ & $1.5(0.6-2.5)$ & 0.10 \\
\hline TBIL onset & $12.7(0.2-22.1)$ & $0.7(0.4-2.1)$ & $0.000 *$ \\
\hline Severity according to Aithal et al. ${ }^{\mathrm{b}}$ & $3(2-4)$ & $2(1-2)$ & $0.002 *$ \\
\hline Jaundice at onset of liver injury (TBIL $>3 \mathrm{mg} / \mathrm{dL}$ ) & $17(81 \%)$ & $0(0 \%)$ & $0.000 *$ \\
\hline MELD at onset of liver injury & $21(6-40)$ & $6(6-10)$ & $0.000 *$ \\
\hline Peak MELD & $23(10-40)$ & $6(6-13)$ & $0.000 *$ \\
\hline Time from onset until peak MELD (days) & $4(0-21)$ & $0(0-77)$ & 0.20 \\
\hline Time from onset until peak ALT & $4(0-99)$ & $0(0-10)$ & 0.012* \\
\hline Time from onset until peak TBIL (days) & $8(0-99)$ & $4(0-77)$ & 0.06 \\
\hline Coagulopathy (INR > 1.5) & $12(57 \%)$ & $0(0 \%)$ & $0.002 *$ \\
\hline Hy's law criteria & $21(100 \%)$ & $4(36 \%)$ & $0.000 *$ \\
\hline Acute liver failure & $7(33 \%)$ & $0(0 \%)$ & $\mathbf{0 . 0 3}^{*}$ \\
\hline Corticosteroid treatment & $7(33 \%)$ & $1(9 \%)$ & 0.13 \\
\hline High-urgency liver transplantation & $2(10 \%)$ & $0(0 \%)$ & 0.23 \\
\hline
\end{tabular}

Categorical variables are presented as number and percentage $[n(\%)]$. Continuous variables are presented as median (range). Laboratory values are presented as $\times$ ULN

$A L P$ alkaline phosphatase, $A L T$ alanine aminotransferase, $A S T$ aspartate aminotransferase, $I N R$ international normalised ratio, $I g G$ immunoglobulin G, MELD Model of End Stage Liver Disease, RUCAM Roussel Uclaf Causality Assessment Method, TBIL total bilirubin, ULN upper limit of normal

*Statistical significance $(p \leq 0.05)$

${ }^{\text {a }}$ Some of the patients only took metamizole as needed and not on a regular daily basis. For those patients, the average daily dose according to the weekly intake was calculated

${ }^{b}$ Aithal et al. Clin Pharmacol Ther. 2011;89(6):806-15

higher (29\% vs $0 \%, p=0.02)$. Regarding histopathological analysis, patients with ALF more frequently presented with mild fibrosis ( $60 \%$ vs $7 \%, p=0.01$ ), while the patients who did not develop ALF showed a higher proportion of eosinophilic cell infiltration (93\% vs $60 \%, p=0.033$; Table 6 of the ESM).

\section{Discussion}

In our cohort of 238 patients with DILI, 32 patients (13\%) were diagnosed with suspected metamizole-induced liver injury. This proportion is in line with the results recently published by Sebode et al., who reported that metamizole 
was the implicated drug in $15 \%$ of their DILI cases [8]. The relatively high percentage of patients using metamizole in our cohort and their DILI cohort can be explained by the growing popularity of metamizole as an analgetic in the German market [3], which emphasises the need to further investigate its potential to cause DILI. In clinical trials, the potential of certain drugs to cause liver injury can be missed owing to the low incidence of overt DILI and therefore might only be revealed by post-marketing surveillance studies or voluntary reporting of suspected cases of liver injury [23, 24]. A DILI diagnosis and causality assessment is challenging, especially in the case of polymedication because no reliable diagnostic test is available. Physicians need to rely on expert opinion and causality assessment tools like the RUCAM score. However, the RUCAM score might be misleading as it comprises previous information on hepatotoxicity and reaction to re-exposure of the implicated drug, which limits its efficacy in evaluating agent-causing liver injury in clinical trials and for drugs commonly not associated with DILI [25]. Thus, the DILI potential of drugs that have not been related to DILI neither in clinical trials nor post-marketing, such as metamizole, can be underestimated. In line with this, only $38 \%$ of the patients in our cohort presented with a RUCAM score for metamizole of 6 or higher while the RUCAM score for the comedication was 6 or above in $14(44 \%)$ cases. We therefore sought to analyse the characteristics of patients with suspected metamizoleDILI to establish a more profound clinical and pathological signature of metamizole-DILI.
Table 5 Characteristics of patients with suspected metamizole-induced liver injury with and without acute liver failure

\begin{tabular}{|c|c|c|c|}
\hline & $\begin{array}{l}\text { Acute liver failure } \\
n=7(22 \%)\end{array}$ & $\begin{array}{l}\text { No acute liver failure } \\
n=25(78 \%)\end{array}$ & $p$ value \\
\hline Age (years) & $35(26-64)$ & $43(19-79)$ & 0.76 \\
\hline Body mass index $\left(\mathrm{kg} / \mathrm{m}^{2}\right)$ & $24.2(18.1-28.3)$ & $23.3(18.9-37.9)$ & 0.66 \\
\hline Female sex & $4(57 \%)$ & $18(72 \%)$ & 0.45 \\
\hline Latency metamizole (days) & $80(27-674)$ & $34(2-370)$ & $\mathbf{0 . 0 3 3} *$ \\
\hline Daily dosage metamizole $(\mathrm{mg})^{\mathrm{a}}$ & $1000(1000-1500)$ & $1500(71-4000)$ & 0.61 \\
\hline RUCAM score for metamizole & $5(3-8)$ & $5(3-9)$ & 0.82 \\
\hline $\begin{array}{l}\text { Maximum RUCAM score for concomi- } \\
\text { tant medication }\end{array}$ & $6(3-9)$ & $5(4-8)$ & 0.66 \\
\hline MH cell test positive for metamizole & $5(56 \%)$ & $19(70 \%)$ & 0.60 \\
\hline $\begin{array}{l}\text { Pattern of injury } \\
\text { Hepatocellular } \\
\text { Mixed } \\
\text { Cholestatic }\end{array}$ & $\begin{array}{l}6(86 \%) \\
1(14 \%) \\
0(0 \%)\end{array}$ & $\begin{array}{l}20(80 \%) \\
4(16 \%) \\
1(4 \%)\end{array}$ & 0.86 \\
\hline Peak ALT & $60.5(14.9-100.0)$ & $33.2(5.9-105.9)$ & 0.09 \\
\hline Peak AST & $50.9(22.5-104.0)$ & $18.9(1.3-162.2)$ & $0.004 *$ \\
\hline Peak ALP & $1.9(1.3-3.4)$ & $1.7(1.0-7.8)$ & 0.40 \\
\hline Peak TBIL & $24.1(20.7-29.9)$ & $4.2(0.5-23.1)$ & $0.000 *$ \\
\hline Peak INR & $2.2(1.7-8.0)$ & $1.2(0.8-2.1)$ & $0.000 *$ \\
\hline MELD at onset of liver injury & $25(20-30)$ & $9(6-26)$ & $0.000 *$ \\
\hline Peak MELD & $26(23-40)$ & $13(6-26)$ & $0.000 *$ \\
\hline Severity according to Aithal et al. ${ }^{\mathrm{b}}$ & $3(3-4)$ & $2(1-3)$ & $0.000 *$ \\
\hline Jaundice (TBIL $>3 \mathrm{mg} / \mathrm{dL})$ & $7(100 \%)$ & $14(56 \%)$ & $\mathbf{0 . 0 3 *}$ \\
\hline Coagulopathy (INR > 1.5) & $7(100 \%)$ & $5(20 \%)$ & $0.000 *$ \\
\hline Hy's law criteria & $7(100 \%)$ & $18(72 \%)$ & 0.11 \\
\hline Corticosteroid treatment & $2(29 \%)$ & $6(24 \%)$ & 0.81 \\
\hline High-urgency liver transplantation & $2(29 \%)$ & $0(0 \%)$ & $0.02 *$ \\
\hline
\end{tabular}

Categorical variables are presented as number and percentage $[n(\%)]$. Continuous variables are presented as median (range). Laboratory values are presented as $\times$ ULN

$A L P$ alkaline phosphatase, ALT alanine aminotransferase, AST aspartate aminotransferase, INR international normalised ratio, MELD Model of End Stage Liver Disease, RUCAM Roussel Uclaf Causality Assessment Method, TBIL total bilirubin, $U L N$ upper limit of normal

*Statistical significance $(p \leq 0.05)$

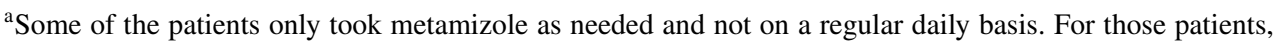
the average daily dose according to the weekly intake was calculated

${ }^{\mathrm{b}}$ Aithal et al. Clin Pharmacol Ther. 2011;89(6):806-15 
Clinical characteristics with a slight female predominance, a median age at onset of 41 years as well as the mostly hepatocellular pattern of injury were comparable to those in patients with metamizole-DILI described recently [8]. Interestingly, it could be shown by thoroughly analysing histopathological features that a relatively large proportion of patients presented with necrosis and eosinophilic cell infiltration ( $85 \%$ respectively).

To identify possible distinguishing features for the effectiveness of causality assessment tools in metamizoleinduced DILI, we performed a subgroup analysis comparing the clinical and histopathological characteristics in patients with probable or highly probably metamizole-DILI (RUCAM $\geq 6$ ) and possible metamizole-DILI (RUCAM 3-5). While ANA positivity was quite a frequent finding in all patients, lower RUCAM scores correlated with AMA positivity. The finding of relatively high ANA and AMA positivity is in line with recent data from our group demonstrating that ANA and also AMA positivity is frequently found in patients with DILI [26]. Furthermore, cholestasis was a prominent feature in the histopathological analysis in patients with higher RUCAM scores; however, this difference did not reach statistical difference. Apart from this, no other relevant differences between patients with higher and lower RUCAM scores were identified. In conclusion, the current results indicate that both patients with RUCAM $\geq 6$ or $<6$ were fairly similar, which argues against the RUCAM score being a reliable discriminating tool in the assessment of metamizole-induced DILI. Thus, in addition to RUCAM, a causality assessment should be based on a variety of methods, for example, expert opinion, evolution of liver parameters after discontinuation of the drug, potential re-exposure and possibly a causality assessment toll such as $\mathrm{MH}$ cell testing. Especially in the case of metamizole, evaluating re-challenges could be useful, as metamizole might be prescribed again even after an episode of DILI because of the underestimation of its DILI potential. In line with this, seven out of the 32 patients from our cohort (22\%) reported a mostly unintentional positive re-challenge with metamizole.

Strikingly, a high proportion of patients developed not only jaundice (66\%) but also ALF (22\%). When focusing on the distinctive features of more severe metamizole-induced DILI cases, the development of clinically relevant jaundice was associated with higher serum transaminases and INR levels at onset and at peak levels, longer latency from the initiation of metamizole treatment, and a more pronounced cholestasis and higher frequency of fibrosis in the histopathological analysis. Jaundiced patients were also more likely to have a more unfavourable outcome with higher proportions of patients developing ALF. Thus, a more severe evolution and possibly the development of ALF needs to be expected in the case of a latency of approximately $2-3$ months from the initiation of metamizole intake as well as severely altered serum liver biochemistry.

Because metamizole has been underestimated as a causative agent in DILI, little is known about the mechanisms of metamizole-induced liver injury. The current understanding of idiosyncratic DILI is that the adaptive immune system plays an important role in the pathogenesis of the liver injury $[6,27]$. The characteristics observed in the patients with suspected metamizole-DILI, i.e., immune cell infiltration in all of the liver specimens with a predominance of eosinophilic cells as well as a high percentage of ANA positivity, indicate that immunological mechanisms might also play a pivotal role in metamizole-induced liver injury. This is in line with scarce previous data on metamizole-DILI proposing a potential immune-mediated mechanism [28].

Our results have major implications for clinical trials as well as post-marketing pharmacovigilance. It could be demonstrated in our cohort that metamizole-induced liver injury needed to be suspected in $13 \%$ of all patients with DILI. Especially in Germany, the use of metamizole has further increased in recent years [3], which might lead to an increase in metamizole-induced DILI cases as well. As metamizole is often taken as a concomitant analgetic medication in polymedicated patients and might also be used by patients participating in clinical trials, it can possibly cause liver injury unrelated to the study medication or other important treatments that cannot easily be replaced by alternative drugs. Because DILI is one of the major reasons for the cessation of drug development and post-marketing withdrawals $[6,9]$, an adequate causality assessment is therefore crucial to avoid unnecessary project terminations [25]. Thus, we encourage physicians treating patients with suspected DILI as well as clinical investigators to consider metamizole as a DILI-causing agent and actively ask the patient about concomitant metamizole use.

Our study has limitations, for instance, the lack of a universal gold standard for the causality assessment. For the current analysis, the DILI diagnosis and causality assessment were based on expert opinion and RUCAM scoring and were supported by $\mathrm{MH}$ cell testing. However, the usefulness of the RUCAM score is limited, as the RUCAM comprises the existing knowledge about the DILI potential of the respective drug. As neither ALF nor elevation of liver function tests is listed in the labelling of metamizole, an underestimation of the actual RUCAM might be possible. In addition, all of the patients had concomitant medications, mainly antibiotics and non-steroidal antirheumatic drugs, which were used in half of the cases. It cannot be fully excluded that the liver injury was caused by the concomitant medication taken by the patients from our cohort. Yet, there is a potential bias, especially regarding the causality assessment based on RUCAM, as liver injury is listed as an adverse reaction in the drug labels of most antibiotics 
and analgesics, which automatically increases the RUCAM score for the majority of the comedications. In line with this, the median RUCAM score for metamizole and for the concomitant drug with the highest RUCAM was 5, which shows that the RUCAM score cannot reliably discriminate metamizole among other possible causative agents of DILI in polymedicated patients. Therefore, we would like to suggest a score of two rather than one in the RUCAM score for metamizole for the item known hepatoxicity. This would automatically lead to higher RUCAM scores and therefore less underestimation of metamizole in DILI. If a score of two for known potential to cause DILI was assumed for metamizole, median RUCAM would have been $6(4-10)$ in our cohort with 21 patients $(66 \%)$ reaching a RUCAM of 6 or higher and 18 of those (85\%) presenting with positive $\mathrm{MH}$ cell test results for metamizole.

A unique strength of our study is the use of an in vitro test system. While in the work of Sebode et al. the DILI diagnosis and causality assessment were solely based on RUCAM scoring [8], in the current analysis, diagnosis was supported by the MH cell test, which can help to identify the causative drug especially in the setting of comedications. The MH cell test showed positive results for metamizole in more than $80 \%$ of patients with a RUCAM for metamizole of 6 or higher and was positive for metamizole in $70 \%$ of the DILI cases with a RUCAM of 3-5 for metamizole. Thus, while a DILI diagnosis and causality assessment solely based on RUCAM may lead to an under- or overestimation of metamizole-DILI, our results including a causality assessment tool further support the role of metamizole as a causative agent in patients with DILI. However, while the MH cell test has been validated in a cohort of patients with positive re-challenge to the respective medication with high sensitivity and specificity [17], external validation has not been completed yet. Thus, the reliability of the MH cell tests as a causality assessment tool needs further assessment. To minimise the overestimation of metamizole-induced DILI by the MH cell test, a variety of control subjects who have been tested with this assay were included (healthy subjects, patients with metamizole intake but liver injury of an alternative cause, patients with DILI without prior metamizole treatment and patients with DILI with prior metamizole but association with a different drug). With the application of results from this collection of samples, the MH cell test would rule out metamizole-induced liver injury with a specificity of $83-94 \%$.

\section{Conclusions}

The identification of 32 patients with suspected metamizole-associated DILI in our cohort of patients with liver injury further underlines the DILI potential of metamizole. Based on our results, the signature of this injury includes a hepatocellular pattern, a high proportion of ANA positivity with also a quite frequent positivity for AMA, and high proportions of inflammatory infiltrates with a predominance of eosinophilic cells and high rates of moderate-to-strong necrosis. A considerable proportion of patients developed jaundice and ALF.

We therefore propose that metamizole should be regarded as a possibly causative agent in DILI, especially in patients with the above-described clinical and histopathological features. The possible causal relationship of metamizole and DILI should also create more awareness among physicians taking the history of previous medications as well as investigators in clinical trials or pharmacovigilance reports. As a future perspective, this could lead to less under-reporting of concomitant use of metamizole by the patient and therefore to a more appropriate consideration of its role in causing DILI.

Supplementary Information The online version contains supplementary material available at https://doi.org/10.1007/s40264-021-01049-z.

Acknowledgements We acknowledge the excellent technical support by Monika Hofstetter.

\section{Declarations}

Funding Open Access funding enabled and organized by Projekt DEAL. Sabine Weber received funding from the Friedrich Baur Foundation. Andreas Benesic received funding from the German Federal Ministry for Economic Affairs and Energy (EXIST grant no. 03EFT9BY56). Andreas Benesic and Alexander L. Gerbes received funding from the Bavarian Ministry of Economic Affairs and Media, Energy and Technology, Germany (m4-award) [grant no. $1330 / 68362 / 34 / 2013]$.

Conflicts of Interest Andreas Benesic has a patent held/filed, is a stock shareholder and had a former management position in MetaHeps $\mathrm{GmbH}$. Alexander L. Gerbes has a patent held/filed and is a stock shareholder of MetaHeps GmbH. The funders had no role in the design of the study; in the collection, analyses or interpretation of data; in the writing of the manuscript, or in the decision to publish the results. Sabine Weber has no conflicts of interest that are directly relevant to the content of this article.

Ethics Approval All procedures were in accordance with the ethical standards of the responsible committee on human experimentation (Faculty of Medicine, LMU Munich; project number 55-13) and with the Declaration of Helsinki.

Consent to Participate Written informed consent was obtained from each patient.

Consent for Publication Not applicable.

Availability of Data and Material All data generated or analysed during this study are included in this published article and its supplementary information files.

Code Availability Not applicable. 
Authors' Contributions All authors contributed to the study conception and design. Material preparation, data collection, analysis and writing of the first draft were performed by Sabine Weber. All authors read and approved the final manuscript.

Open Access This article is licensed under a Creative Commons Attribution-NonCommercial 4.0 International License, which permits any non-commercial use, sharing, adaptation, distribution and reproduction in any medium or format, as long as you give appropriate credit to the original author(s) and the source, provide a link to the Creative Commons licence, and indicate if changes were made. The images or other third party material in this article are included in the article's Creative Commons licence, unless indicated otherwise in a credit line to the material. If material is not included in the article's Creative Commons licence and your intended use is not permitted by statutory regulation or exceeds the permitted use, you will need to obtain permission directly from the copyright holder. To view a copy of this licence, visit http://creativecommons.org/licenses/by-nc/4.0/.

\section{References}

1. Andrade S, Bartels DB, Lange R, Sandford L, Gurwitz J. Safety of metamizole: a systematic review of the literature. J Clin Pharm Ther. 2016;41(5):459-77.

2. Leone R, Conforti A, Venegoni M, Motola D, Moretti U, Meneghelli I, et al. Drug-induced anaphylaxis: case/non-case study based on an Italian pharmacovigilance database. Drug Saf. 2005;28(6):547-56.

3. Huber M, Andersohn F, Sarganas G, Bronder E, Klimpel A, Thomae M, et al. Metamizole-induced agranulocytosis revisited: results from the prospective Berlin case-control surveillance study. Eur J Clin Pharmacol. 2015;71(2):219-27.

4. Bjornsson ES. Liver injury associated with the analgetic drug metamizole. Br J Clin Pharmacol. 2020;86(7):1248-50.

5. Lutz M. Metamizole (dipyrone) and the liver: a review of the literature. J Clin Pharmacol. 2019;59(11):1433v42.

6. Kullak-Ublick GA, Andrade RJ, Merz M, End P, Benesic A, Gerbes AL, et al. Drug-induced liver injury: recent advances in diagnosis and risk assessment. Gut. 2017;66(6):1154-64.

7. Raschi E, De Ponti F. Drug-induced liver injury: towards early prediction and risk stratification. World J Hepatol. 2017;9(1):30-7.

8. Sebode M, Reike-Kunze M, Weidemann S, Zenouzi R, Hartl J, Peiseler M, et al. Metamizole: an underrated agent causing severe idiosyncratic drug-induced liver injury. Br J Clin Pharmacol. 2020;86(7):1406-15.

9. Real M, Barnhill MS, Higley C, Rosenberg J, Lewis JH. Druginduced liver injury: highlights of the recent literature. Drug Saf. 2019;42(3):365-87.

10. Senior JR. Evolution of the Food and Drug Administration approach to liver safety assessment for new drugs: current status and challenges. Drug Saf. 2014;37(Suppl. 1):S9-17.

11. Rochon J, Protiva P, Seeff LB, Fontana RJ, Liangpunsakul S, Watkins PB, et al. Reliability of the Roussel Uclaf Causality
Assessment Method for assessing causality in drug-induced liver injury. Hepatology. 2008;48(4):1175-83.

12. Aithal GP, Watkins PB, Andrade RJ, Larrey D, Molokhia $\mathrm{M}$, Takikawa $\mathrm{H}$, et al. Case definition and phenotype standardization in drug-induced liver injury. Clin Pharmacol Ther. 2011;89(6):806-15

13. Bénichou C. Criteria of drug-induced liver disorders. J Hepatol. 1990;11(2):272-6.

14. Danan G, Benichou C. Causality assessment of adverse reactions to drugs. I. A novel method based on the conclusions of international consensus meetings: application to drug-induced liver injuries. J Clin Epidemiol. 1993;46(11):1323-30.

15. Hayashi PH. Drug-induced liver injury network causality assessment: criteria and experience in the United States. Int J Mol Sci. 2016;17(2):201.

16. Benesic A, Leitl A, Gerbes AL. Monocyte-derived hepatocyte-like cells for causality assessment of idiosyncratic drug-induced liver injury. Gut. 2016;65(9):1555-63.

17. Benesic A, Rotter I, Dragoi D, Weber S, Buchholtz ML, Gerbes AL. Development and validation of a test to identify drugs that cause idiosyncratic drug-induced liver injury. Clin Gastroenterol Hepatol. 2018;16(9):1488-94.e5.

18. Tillmann HL, Suzuki A, Barnhart HX, Serrano J, Rockey DC. Tools for causality assessment in drug-induced liver disease. Curr Opin Gastroenterol. 2019;35(3):183-90.

19. LiverTox: clinical and research information on drug-induced liver injury. Bethesda (MD): National Institute of Diabetes and Digestive and Kidney Diseases. 2012. https://www.ncbi.nlm.nih.gov/ books/NBK547852/. Accessed 12 Dec 2020.

20. Benesic A, Rahm NL, Ernst S, Gerbes AL. Human monocyte-derived cells with individual hepatocyte characteristics: a novel tool for personalized in vitro studies. Lab Invest. 2012;92(6):926-36.

21. Polson J, Lee WM. AASLD position paper: the management of acute liver failure. Hepatology. 2005;41(5):1179-97.

22. Regev A, Bjornsson ES. Drug-induced liver injury: morbidity, mortality, and Hy's law. Gastroenterology. 2014;147(1):20-4.

23. Kaplowitz N. Drug-induced liver disorders: implications for drug development and regulation. Drug Saf. 2001;24(7):483-90.

24. Raschi E, De Ponti F. Drug- and herb-induced liver injury: progress, current challenges and emerging signals of post-marketing risk. World J Hepatol. 2015;7(13):1761-71.

25. Regev A, Seeff LB, Merz M, Ormarsdottir S, Aithal GP, Gallivan $\mathrm{J}$, et al. Causality assessment for suspected DILI during clinical phases of drug development. Drug Saf. 2014;37(Suppl. 1):S47-56.

26. Weber S, Benesic A, Buchholtz ML, Rotter I, Gerbes AL. Antimitochondrial rather than antinuclear antibodies correlate with severe drug-induced liver injury. Dig Dis. 2020. https://doi.org/ $10.1159 / 000511635$.

27. Mosedale M, Watkins PB. Drug-induced liver injury: advances in mechanistic understanding that will inform risk management. Clin Pharmacol Ther. 2017;101(4):469-80.

28. Herdeg C, Hilt F, Buchtemann A, Bianchi L, Klein R. Allergic cholestatic hepatitis and exanthema induced by metamizole: verification by lymphocyte transformation test. Liver. 2002;22(6):507-13. 